\title{
CONSEQUENCES OF MODELING DEMANDS ON NUMERICAL ROCKET THRUST CHAMBER FLOW SIMULATION TOOLS
}

\section{O. Knab, H. Riedmann, B. Ivancic, C. Höglauer, M. Frey, and T. Aichner}

Airbus DS GmbH

Munich 81663, Germany

\begin{abstract}
Numerical simulation of liquid rocket thrust chamber flows is a challenging task which requires a comprehensive tool validation strategy encompassing laboratory- to full-scale test cases. While the former are widely used as the first step to verify advanced numerical schemes and thermochemical models, the latter can often no more be simulated in a reasonable time frame due to the extreme computational effort necessitated by the enlarged dimensions and configurational changes. Conclusions drawn from tools with such limitations are only of little help for a thrust chamber designer and, hence, ill-posed to adequately tackle the simulation challenges of such a device. This paper discusses the modeling demands for numerical thrust chamber flow simulation tools and outlines the indispensable validation approach from laboratory- via subto full-scale configurations using consistent model features throughout the test cases. Consequences of this obligation are exposed to drive the tool setup. Exemplarily, the Airbus DS thrust chamber flow simulation philosophy is presented.
\end{abstract}

\section{INTRODUCTION}

An Ariane 5 mission supplying the International Space Station (ISS) with the European Automated Transfer Vehicle (ATV) operates around 60 rocket thrusters which differ in function (launcher propulsion, roll control, booster separation, attitude control, and payload maneuvering), thrust class $(0.2-5300 \mathrm{kN})$, and type (solid, liquid mono-, and bipropellant) (Fig. 1). In particular, the liquid engines work with diverse propellants such as hydrazine, monomethylhydrazine (MMH), and hydrogen as fuel and dinitrogen tetroxide (NTO) and oxygen as oxidizer fea- 


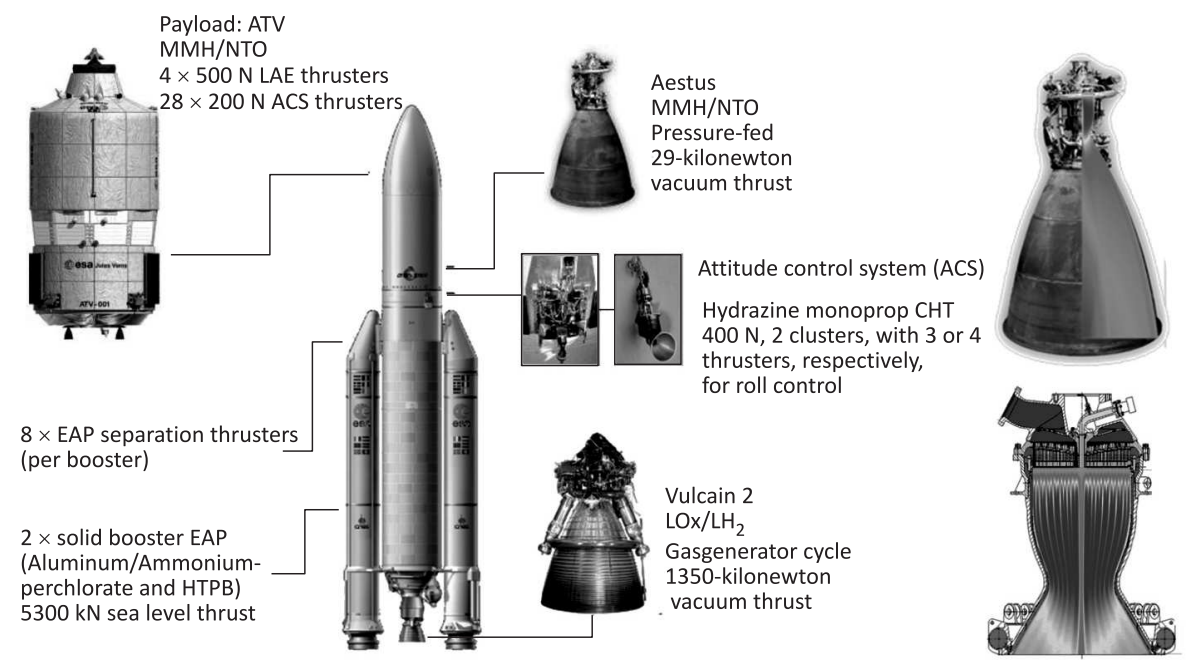

Figure 1 Rocket thrusters operated on Ariane 5 during an ISS supply mission with ATV (left) and Airbus DS combustion and heat transfer simulations of the Aestus and Vulcain 2 thrust chambers (right)

turing distinct fluid characteristics (cryogenic or storable) and requiring different propellant injection technologies.

Airbus DS as the leading European developer and manufacturer of liquid rocket thrust chambers has acquired diverse engineering competencies over the last 40 years encompassing the sizing and contouring of combustion chambers and nozzle extensions, the definition of injector patterns and element configurations, the evaluation of heat transfer into cooling circuits and injector face plates as well as the tailoring of cooling channels and wall thickness distributions to fulfill specified performance, pressure budget, and life requirements [1, 2 ]. Key success factors are reliable computational tools for efficient layout and optimization of the respective thrust chamber components. Their validation is a long-term process and comprises the mapping of numerous test cases ranging from laboratory- up to full-scale under flight conditions (Fig. 2). Hence, the selection of the tools is governed by the ability to cover this extreme dimensional and operational range nonetheless mastering the inherent thermochemical complexities.

For the design and optimization of liquid rocket thrust chambers, the use of numerical flow simulation tools is a promising approach to reduce test and redesign efforts and, by that development time and cost. In general, the requirements for such computational fluid dynamics (CFD) tools are fairly simple, namely, to accurately predict: 


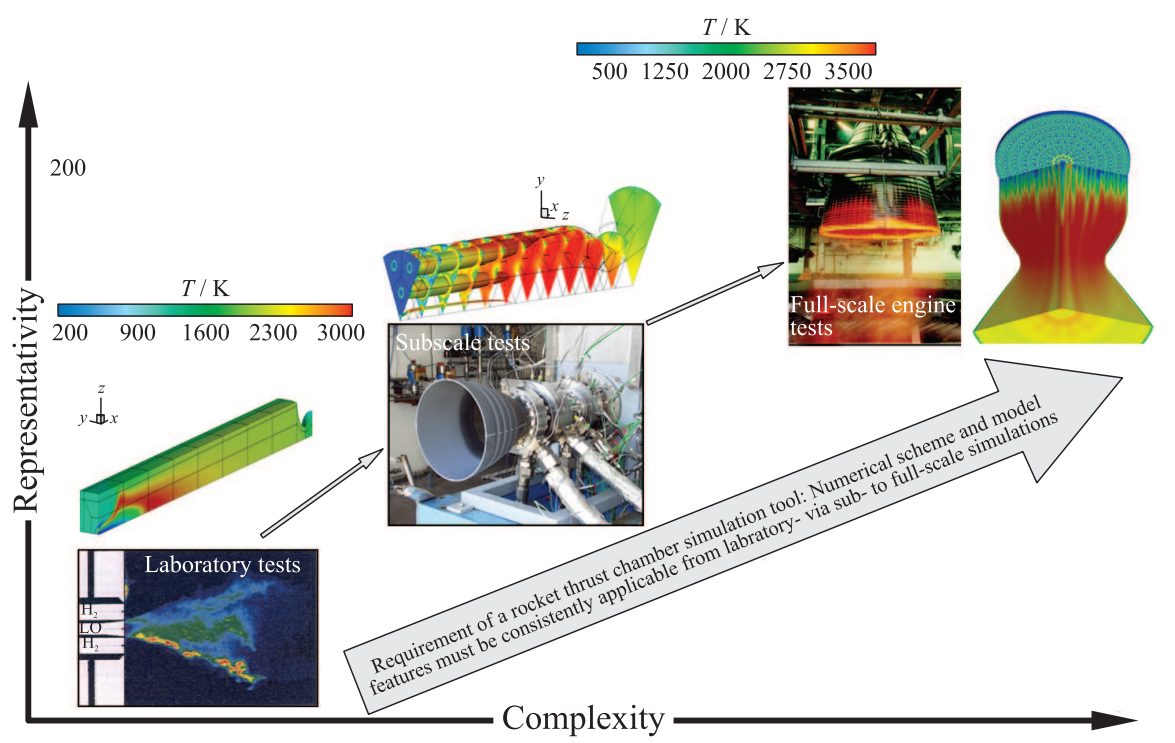

Figure 2 As for the hardware, a thrust chamber flow simulation tool has to demonstrate its technology readiness level by increasing model complexity and hence representativeness up to full-scale

R1 the transformation of chemical energy into thermal energy (i. e., the combustion efficiency in the combustion chamber) and, further downstream in the nozzle extension, the transformation of thermal energy into kinetic energy, both determining the performance of the thrust chamber;

$\mathbf{R 2}$ the local heat transfer into the thrust chamber wall in order to manage heat load and life-time requirements properly; and

R3 to deliver results in time frames short enough to allow actual design and optimization work (e.g., 1-3 days in conceptual phase, 1-3 weeks in design and optimization phases, 1-3 months in justification phase).

It is emphasized that R3 includes the capability to efficiently assess the thrust chamber behavior in its predefined operational envelope, i. e., chamber pressure (or mass flow rate) over mixture ratio box. In order to fulfill these requirements, classical Reynolds-averaged Navier-Stokes (RANS), unsteady RANS (URANS), detached eddy simulation (DES), or more and more upcoming large eddy simulation (LES) solvers have to be extended by relevant models. The major thermochemical phenomena prevailing in liquid rocket thrust chambers which have to be reproduced by the tools in a balanced manner are: 
P1 propellant disintegration and mixing at sub- and supercritical chamber pressures;

P2 turbulent combustion of different propellant combinations such as liquid oxygen (LOx) / hydrogen, LOx/methane, and MMH/NTO;

P3 turbulent heat transfer accounting for wall roughness and condensation at overcooled walls; and

$\mathbf{P} 4$ heat removal such as by film and regenerative cooling.

Since all aforementioned phenomena strongly influence requirements $\mathrm{R} 1$ and $\mathrm{R} 2$, a tool package encompassing a variety of models is needed. Numerical flow simulation tools which do not incorporate the full range of models or do not fulfill requirement R3 will, hence, not be able to support the design process of liquid rocket thrust chambers. Apparently, this has severe consequences on tool configuration and its validation strategy. With other words, tools and methods which can only be applied to laboratory-scale test cases are neither oriented towards the simulation of operating rocket propulsion systems as depicted in Fig. 1 nor conclusive for tool developments with such focus.

This paper outlines the essential steps for a numerical thrust chamber flow simulation tool to achieve the necessary validation level before providing a benefit for real hardware layout. In this connection, main emphasis is put on hot gas side modeling (P1-P3), however, having in mind that structural cooling techniques (P4) determine the wall temperature level and, hence, the thermal boundary conditions. Usually, single-element combustors are simulated to first manage nonpremixed propellant disintegration and combustion. As illustrated in Fig. 2, the representativeness is quite low but also rather complex. Often, either the mixture ratio or the chamber pressure is kept low to avoid active cooling of the test specimen. Consequently, phenomena P1 to P4 are different compared to the ones occurring in multielement configurations and the requirements $\mathrm{R} 1$ and $\mathrm{R} 2$ cannot be demonstrated. Nevertheless, first conclusions on necessary mesh resolution, turbulent combustion model settings, and expensed computational time can be deduced from these simulations by comparison to experimental data. However, such simulations have, by far, not demonstrated the tool's grade of a numerical thrust chamber design supportive device.

Transferring such elaborated mesh resolution requirements and model parameter settings to a multielement injector case is the next building block of a tool development strategy. On this so-called subscale combustor level, element-toelement and element-to-wall interaction mechanisms have to be resolved yielding representative values for combustion efficiency and wall heat flux distribution. Furthermore, cooling methods such as film or regenerative cooling have to be 
taken into account to capture the wall boundary conditions accurately. This implies a fluid-structure coupling including heat removal via coolant flow or radiation. Normally, subscale combustion devices make use of full-scale injector elements arranged in a smaller package, however, being representative in crucial characteristics such as element mass flow loading, element-to-element distance, and element-to-wall distance. Consequently, phenomena P1 and P2 are almost perfectly and phenomena $\mathrm{P} 3$ and $\mathrm{P} 4$ largely reproduced so that the mastering of subscale test cases are the baseline for any rocket thrust chamber simulation tool development.

Only numerical tool packages which feature this validation level can be applied to full-scale liquid rocket thrust chamber engineering and thus contribute to reduce hardware development time and cost. A key, however, is still the fulfillment of requirement R3: Numerical solver inherent mesh resolution requirements derived from single element and/or subscale multielement combustor simulations must allow the transfer to a full-scale simulation without breaking R3. Otherwise, the numerical method is not applicable in a development phase and conclusions drawn from its laboratory-scale simulations are not pointing the way ahead. Figure 2 illustrates the path a rocket thrust chamber hardware and design supportive simulation tool has to go in order to become operation relevant. Since up to now no full-scale and only some restricted subscale rocket thrust chamber simulations have been presented in the open literature using URANS, DES, or LES solvers, the design and optimization task and, herewith, the envisaged development cost reduction has still to be done by RANS solvers. On the basis of single element test case computations, the latter, however, are sometimes argued to be inferior to URANS and LES simulations.

The subsequent sections will disprove this statement and outline that a single flame computation capability is only a small part in the development of a rocket thrust chamber flow simulation tool. The Airbus DS modeling philosophy is presented and shown to fulfill the addressed model consistency up to full-scale simulations.

\section{SINGLE ELEMENT COMBUSTOR TEST CASES}

Due to their simplicity and offer of progressive measurement data, single injector tests are often used as the first step to verify numerical simulation tools which have the target to finally master combustion and heat transfer computations in rocket combustion devices. Favored are the gaseous oxygen $\left(\mathrm{GO}_{2}\right)$ / gaseous hydrogen $\left(\mathrm{GH}_{2}\right)$ PennState combustor [3], a single coaxial injector fed with oxidizer-rich and fuel-rich preburner gases and ONERA's Mascotte single element combustor [4], burning $\mathrm{GH}_{2}$ and $\mathrm{GO}_{2}$ at subcritical (10 bar) and su- 
percritical (60 bar) pressure conditions. In the following two subsections, the meaning of these test cases is outlined and results available in the literature are discussed. From these simulations, conclusions are drawn and consequences for multielement configuration calculations are derived.

\subsection{PennState Test Case}

The so-called $\mathrm{GO}_{2} / \mathrm{GH}_{2}$ PennState combustor is operated at the Cryogenic Combustion Laboratory at the Pennsylvania State University. The operating conditions are adjusted to full flow staged combustion (FFSC) engine cycles which burn the liquid propellants in two preburners (oxidizer-rich and fuel-rich) and feed their hot exhaust gases into the main combustion chamber. From modeling point of view, the gaseous injection conditions are easy to handle and no models are needed to capture cryogenic propellant characteristics and the related more complex disintegration process. The test case was presented at the 3rd International Workshop on Rocket Combustion Modeling in 2006 [5] and aligned to characterize the chamber wall heat flux distribution.

Subsequent to the workshop, many numerical tool developers have simulated this test case and discussed their results in the literature, among them are Tucker et al. in 2008 [6], Sozer et al. [7], Daimon et al. [8], Lian and Merkle in 2010 [9], Lempke et al. in 2011 [10], and Ivancic et al. in 2015 [11]. While Lempke et al., Lian and Merkle, and Tucker et al. concluded that any credible test case simulation must be time accurate (Fig. 3), Sozer et al., Daimon et al., and Ivancic et al. (Fig. 4) displayed in contrast that RANS simulations are capable to reflect the measurement data fairly well. Tucker et al. addressed that the computational effort increases by a factor of 15 switching from an axisymmetric RANS computation to an axisymmetric URANS simulation. Recognizing a two-dimensional (2D) axisymmetric approach as shortcoming of an inherently three-dimensional (3D) unsteady flow leads to a factor of 150 (another factor of 10) in increased computation time for a 3D unsteady flow computation [6]. Having the mission of Fig. 2 as a target, it appears worthwhile to understand why and how RANS simulations can master the test case.

A steady flow simulation, of course, cannot resolve unsteady features of a coaxial injector flame, which are mainly triggered by the vortex flow behind the injector post tip where the flame anchors to the injector. Demonstrating grid independence in that tiny domain of the combustor is infeasible for RANS solvers since the solution fails to converge as soon as certain mesh resolution is exceeded. Nevertheless, a sufficient resolution is necessary to capture the turbulence production in that region, which again governs propellant mixing and combustion further downstream, and grid convergence can be demonstrated up to a definite fineness. The post tip spans around $0.5 \mathrm{~mm}$ in radial direction and 


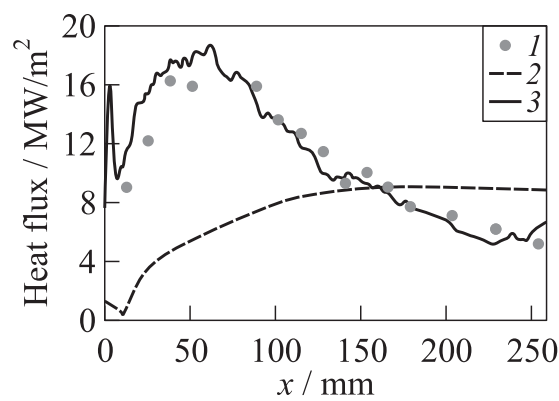

(a)

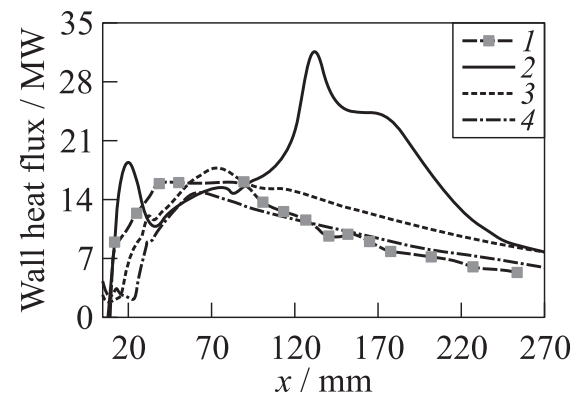

(b)

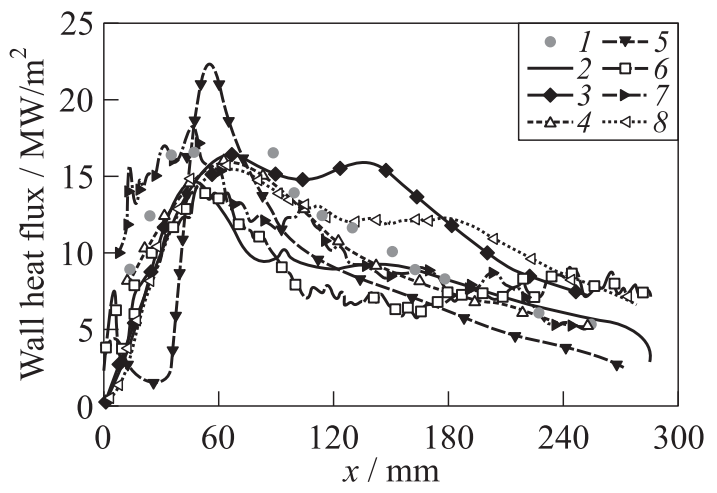

(c)

Figure 3 Comparison of PennState single injector test case simulation results: while Lian and Merkle [9] (a) (1 - experiment; 2 - steady; and 3 - time-averaged unsteady) and Lempke et al. [10] (b) (1 - experiment; 2 - RANS 2nd order; 3 URANS 2nd order; and 4 - URANS 5th order) concluded that RANS simulations are not able to reflect the measured heat flux profile properly, Tucker et al. [6] (c) (1Pal et al. [5]; $2-1 ; 3-6 ; 4-$ LES (SNL); 5 - LES (GIT); 6 - LES (PSU); 7 - URANS (Purgue); and 8 - URANS (Marshall Space Flight Center, MSFC)) did not find a monotonic convergence to the test data with increasing computational tool fidelity

is quite nonuniformly resolved by the tool developers: 11 to 96 grid cells are used for LES computations [6], 70 [6] to 80 [10] for URANS simulations and 16 [11] to 70 [8] for RANS calculations. Obviously, also unsteady computations succeed on different mesh resolutions. At this place, it is reminded that a consistent model transfer from single to multielement configurations requires the retention of the post tip resolution.

Grid resolution is, however, not the only parameter to be checked when generating valuable solutions. Because there are no universal figures valid, also the 


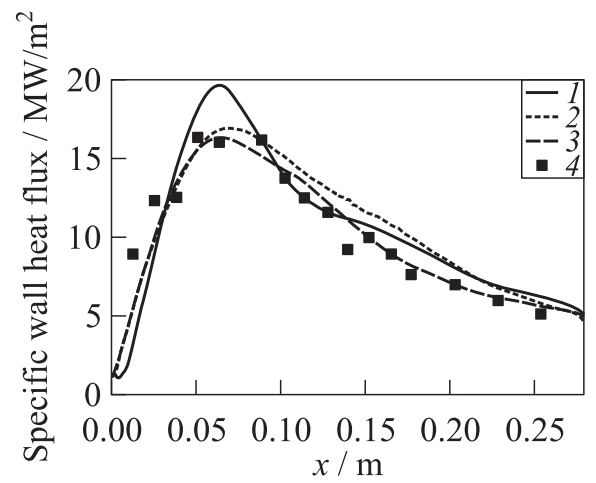

Temperature / K

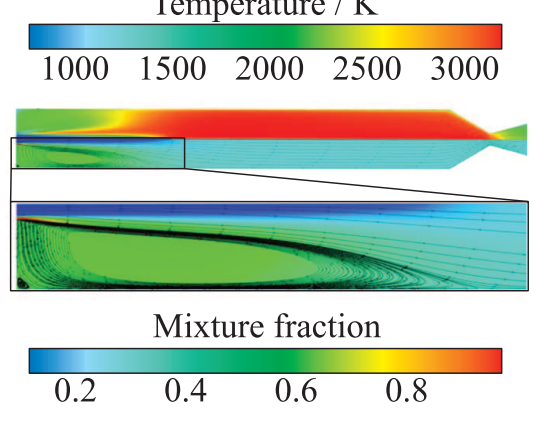

(a)

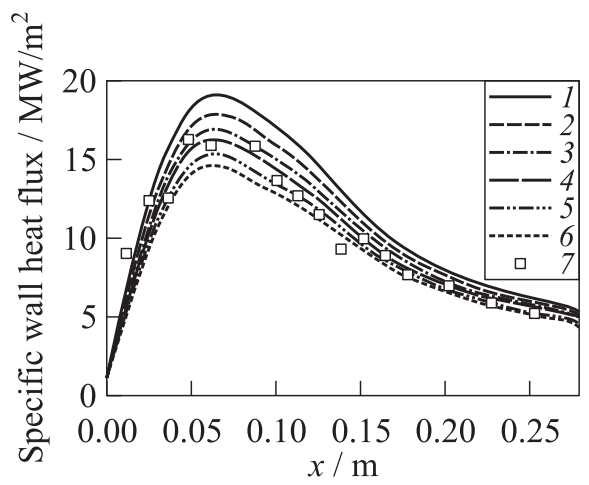

(b)

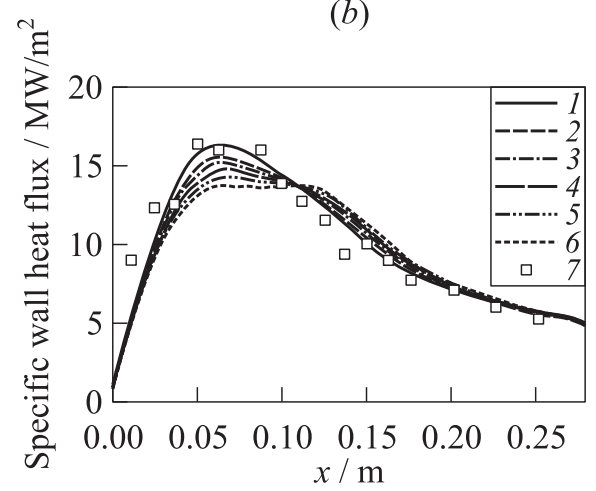

(c)

Figure 4 Wall heat flux distributions obtained for the PennState single element injector from diverse RANS simulations $(a)$ ( 1 - TAU; 2 - CFX Flamelet; 3 Rocflam3; and 4 - test data) and sensitivity study [11] on turbulent Prandtl number $\left(1-\operatorname{Pr}_{t}=0.6 ; 2-0.7 ; 3-0.8 ; 4-0.9 ; 5-1.0\right.$; and $\left.6-\operatorname{Pr}_{t}=1.1\right)$ at $\mathrm{Sc}_{t}=0.6$ $(7-$ experiment $)(b)$ and turbulent Schmidt number settings $\left(1-\mathrm{Sc}_{t}=0.6 ; 2-\right.$ $0.7 ; 3-0.8 ; 4-0.9 ; 5-1.0$; and $\left.6-\mathrm{Sc}_{t}=1.1\right)$ at $\operatorname{Pr}_{t}=0.9(7-$ experiment $)(c)$

turbulent Schmidt $\left(\mathrm{Sc}_{t}\right)$ and Prandtl $\left(\mathrm{Pr}_{t}\right)$ numbers have to be predefined in all types of numerical simulations (except for in direct numerical simulation (DNS)). While the former influences turbulent mixing, the latter drives turbulent heat conduction. However, none of the above mentioned publications addresses the impact of these parameter settings, except for [11].

Figure 4 displays a RANS sensitivity study of $\mathrm{Sc}_{t}$ and $\mathrm{Pr}_{t}$ settings on the wall heat flux profile. Not any of the numbers is wrong; nevertheless, a distinct impact on the heat flux amount and profile is visible. Consequently, the RANS tendency to underestimate turbulence and mixing intensity can, to a certain amount, be counteracted by the setting of these parameters. This is also cred- 
ible in LES via the small-scale submodels, wherein $\mathrm{Sc}_{t}$ and $\operatorname{Pr}_{t}$ numbers are, of course, different to the ones in RANS and URANS since they merely model the transport of small-scale eddies.

As indicated in Fig. 4, the main challenge of this test case is the correct resolution of the huge recirculation zone generated by the large element-to-wall distance. Such vortex flow imposes losses and is usually not present to such an extent in real rocket thrust chambers using coaxial injectors. In order to limit the simulated turbulent viscosity increase in the vicinity of the reattachment point, dedicated models may be necessary (see [11]). In nonreacting flows, the reattachment point would characterize the highest heat flux peak. In reacting flows, however, the local heat conductivity is determined by the local gas composition which varies along the wall. Since the vortex transports water vapor into the fuel-rich gas entering the chamber through the outer injector annulus, the mixture ratio increases along the wall, whereas the heat conductivity decreases. The latter leads to the heat flux peak located upstream of the reattachment point.

As a conclusion, this test case demonstrates impressively that the tool package encompassing solver, grid, turbulence, and chemistry models as well as the respective flow and model parameters is crucial and not a specific attribute such as, for example, a steady or unsteady solver. Apparently, also RANS packages can properly reflect the test data, even better than improper URANS or LES packages.

Since FFSC cycle engines are not in the perimeter of European launcher propulsion development roadmaps, the investigated operational conditions are not at all representative for the Ariane rocket thruster scenario shown in Fig. 1. Moreover, the PennState combustor's characteristic length of $l^{*}=6.5 \mathrm{~m}$ and contraction ratio of $\varepsilon_{c}=21.6$ are unrealistically large for real rocket combustion devices. Accordingly, the Mach number within the combustor is $\mathrm{Ma}=0.03$ and far too low to reflect phenomena P1 (propellant disintegration), P2 (combustion), and P3 (heat transfer) at actual conditions. In addition, the specified preburner gas inflow conditions seem not to be compatible with the given chamber pressure, as discussed in [11], and thus, the tools' capability to reflect the combustion efficiency (R1) cannot conclusively be evaluated. The ability to satisfactorily simulate this test case should, therefore, only be seen as entry point for any thrust chamber flow simulation tool.

\subsection{Mascotte A-10, A-60, and C-60 Test Cases}

The Mascotte single element combustor is operated by ONERA in Palaisseau, France [4]. The chamber has a square cross section with an edge length of $50 \mathrm{~mm}$ and is equipped with helium-cooled quartz windows on two sides for optical access. Three test cases, A-10, A-60, and C-60, have been reported in the litera- 
ture burning $\mathrm{GH}_{2}\left(T_{\mathrm{fu}}=287 \mathrm{~K}\right)$ and cryogenic oxygen $\left(T_{\mathrm{ox}}=85 \mathrm{~K}\right)$ via a shear coaxial injector at 10 - and 60 -bar chamber pressure, respectively. The propellant conditions are flight hardware relevant since at 10 bar, the oxygen is liquid, as, e.g., in the Ariane 5 upper stage engine HM7, and at 60 bar transcritical, as, e. g., in its main stage engine Vulcain 2. However, the operated mixture ratios are between 1.4 and 2.2 and, therefore, neither representative for gas generator combustion $(\mathrm{O} / \mathrm{F}<1)$ nor for main chamber combustion $(\mathrm{O} / \mathrm{F}>5)$. As for the PennState combustor, the characteristic length $l^{*}=6.6 \mathrm{~m}$ and the contraction ratio $\varepsilon_{c}=14.1$ are again unrealistically large to closely reflect actual phenomena P1 to P3. Nevertheless, the test cases are very attractive for tool developers since they cover sub- and transcritical oxygen injection conditions. It is reminded here that the future Ariane 6 upper stage expander cycle engine VINCI will operate in both regimes, which means that a simulation tool should also be able to compute both test cases.

The Mascotte A-10 10-bar test case has been described in detail by Vingert and Habiballah [12]. The experimental data which are used for comparison with numerical results originate from two publications: there is an Abel-transformed emission image and mean temperature profiles published by Candel et al. [13] as well as additional mean temperature profiles published by Grisch et al. [14]. The LOx jet disintegration may be simulated by a strict Eulerian two-phase approach or an Euler-Lagrange method, which prescribes a sparse distribution of LOX droplet parcels and traces them while evaporating on their path through the continuous gas flow. Most of the simulations found in literature make use of the Eulerian-Lagrangian approach, among them are Nicole et al. in 2009 [15], Lempke et al. [10], Izard and Mura in 2011 [16], Ivancic et al. in 2012 [17], and Riedmann et al. in 2013 [18]. A representative for the strict Eulerian approach, which treats multiphase flows as coexisting continua in each phase and resolves the primary LOx jet atomization, is Farmer et al. in 2005 [19]. All of the aforementioned contributions provided RANS solutions, including Lempke et al., who concluded yet from the PennState combustor simulation that RANS is infeasible to reflect the single element flow physics correctly. Although this overview is not complete, it becomes evident that whenever the physics become more complex, even already for a single element flow simulation, the necessity of an unsteady computation seems to fade into the background.

Since the Mascotte chamber has a square cross section, it is well suited to check $2 \mathrm{D}$-axisymmetric vs. $3 \mathrm{D}$ computations. Moreover, the impact of spray initialization can be evaluated. In the test case description, it is proposed to initialize the oxygen spray along a solid cone protruding into the chamber and approximating in that way the LOx core. As can be seen from Fig. 5, Izard and Mura [16] as well as Ivancic et al. [17] successfully employed this procedure in 2D-axisymmetric simulations. As for the PennState coaxial injector, the flame is attached close to the injector exit. Lempke et al. [10] and Nicole et al. [15] applied this approach also in 3D, where LOx droplet 


\begin{tabular}{rllll}
\multicolumn{5}{c}{$T / \mathrm{K}$} \\
\hline $500 \quad 688$ & 1250 & 2188 & 3500
\end{tabular}

\begin{tabular}{|cccccc|}
\multicolumn{8}{c}{$T / \mathrm{K}$} \\
\hline 500 & 1000 & 1500 & 2000 & 2500 & 3000
\end{tabular}
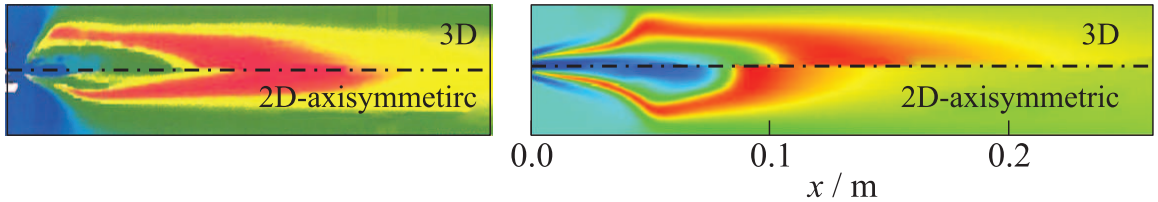

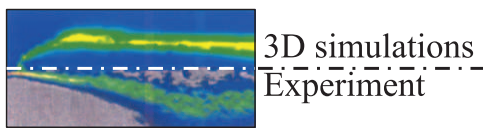

(a)

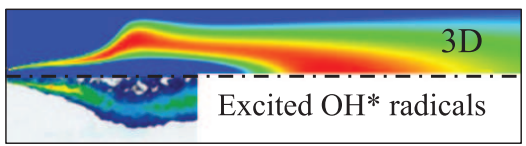

(b)

Figure 5 Comparison of Mascotte 10-bar test case simulations of Nicole et al. [15] (a) and Riedmann et al. [20] (b): Predicted change in flame shape when switching from 2D-axisymmetric to 3D computation and comparison to experimental image of excited $\mathrm{OH}^{*}$ radicals (median plane images)

parcels were initiated at various locations along the cone (see illustration in Fig. 5). Since from grid generation point of view this procedure may become sophisticated in a subscale or even full-scale multiinjector configuration, Riedmann et al. [18] simplified the procedure and injected the LOx droplets solely from the face plate into the combustion chamber. By this method, the grid must not be adapted to the injector position, instead, as displayed in Fig. 5, respective source terms reflecting the propellant inflow characteristics are set across the inflow areas. This strategy has been successfully applied by Airbus DS for a very long time [1], but firstly here transferred into a 3D computation. Notice that with this methodology, different injector patterns can be simulated for multiinjector configuration optimization without changing the grid.

Since comparisons of $3 \mathrm{D}$ and 2D-axisymmetric computations have so far only been presented by Nicole et al. [15] and Riedmann et al. [20], their results are briefly discussed here. As can be seen from Fig. 6, Nicole et al. predicts in 3D a flame shortening, while Riedmann et al. a flame elongation. From the plots, it appears that Nicole et al. used the square edge length as diameter for the axisymmetric simulation and, hence, reduced the chamber cross section, while Riedmann et al. slightly enlarged the diameter to maintain the cross section and, hence, the chamber Mach number. However, only the latter matches the $\mathrm{OH}^{*}$ emission image fairly well. It should also be mentioned that for test data reduction via Abel-transformation, an axisymmetric flame has been assumed, 


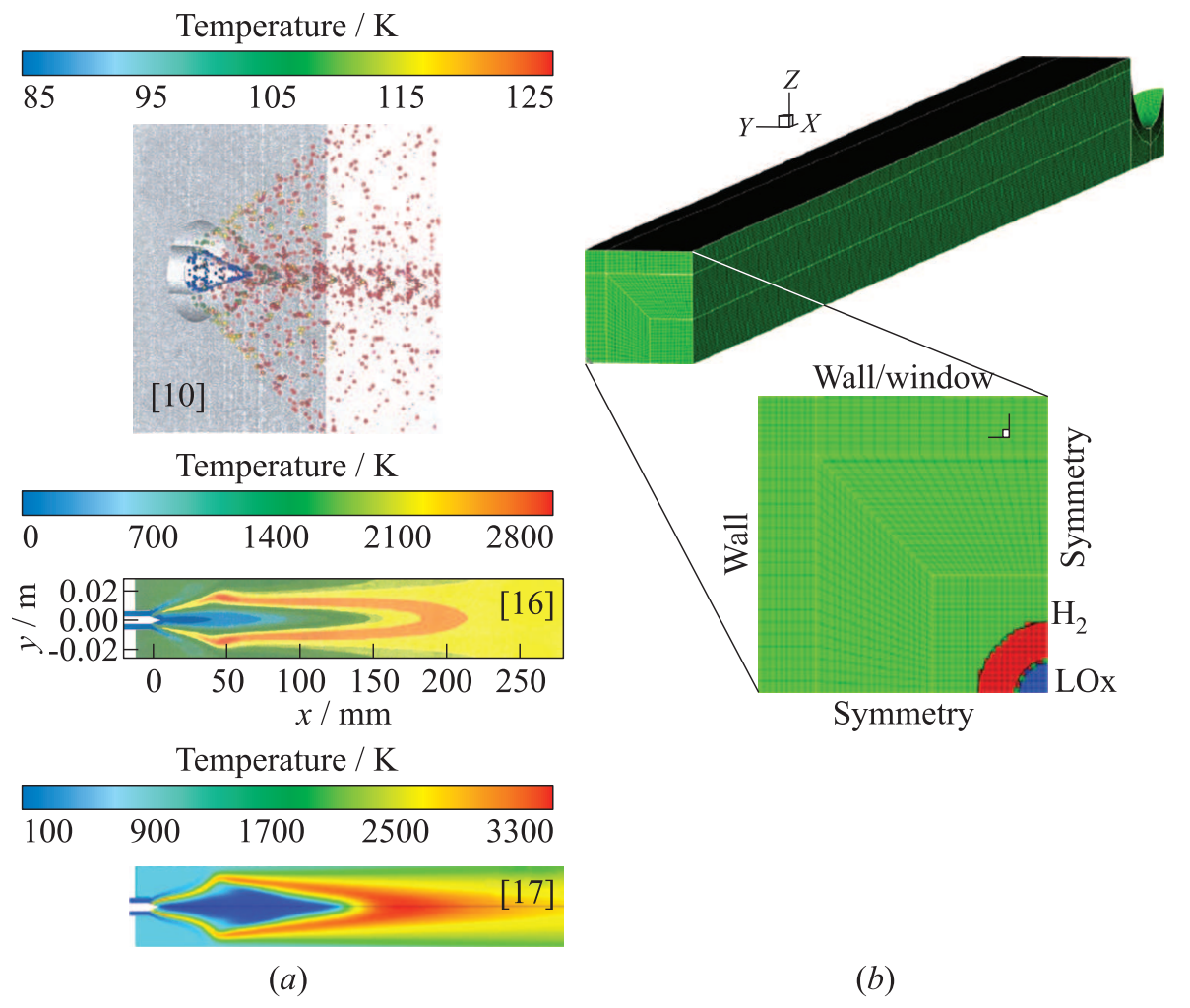

Figure 6 Different types of LOx spray initialization methods: Along a solid cone protruding into the chamber as applied by Lempke et al. [10], Izard and Mura [16] and Ivancic et al. [17] (a) and as proposed by Riedmann et al. [18] over the area on the face plate, where LOx flows into the chamber $(b)$

while both $3 \mathrm{D}$ simulations reveal a distinct nonaxisymmetric flame. In any case, the simplified spray initialization procedure applied by Riedmann et al. appears to be suitable. This is further confirmed by the comparison of simulated temperature profiles and discrete CARS measurements shown in Fig. 7. The predicted temperatures along the $x$-axis at $z=0 \mathrm{~mm}$ and various heights $(y=0$, 5,10 , and $15 \mathrm{~mm}$ ) match fairly well with the measured temperatures. Also, the results obtained from a $2 \mathrm{D}$-axisymmetric simulation reproduce the measurement data satisfactorily. There is no example in literature known demonstrating that URANS or LES solvers compute this test case more accurately than the RANS solvers.

The Mascotte 60 -bar test case has first been introduced by Habiballah and Zurbach [21] and is referenced as A-60. The used shear coaxial injector had no 


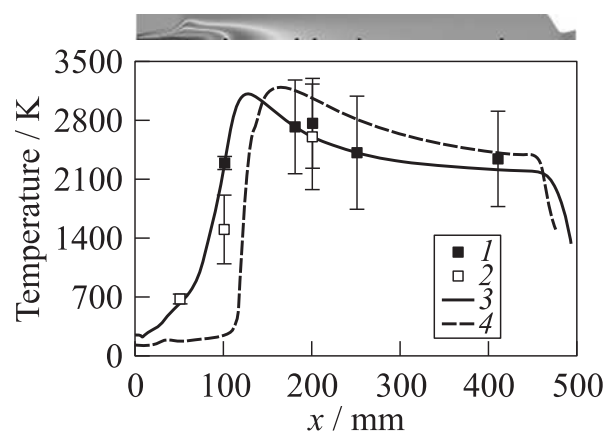

(a)

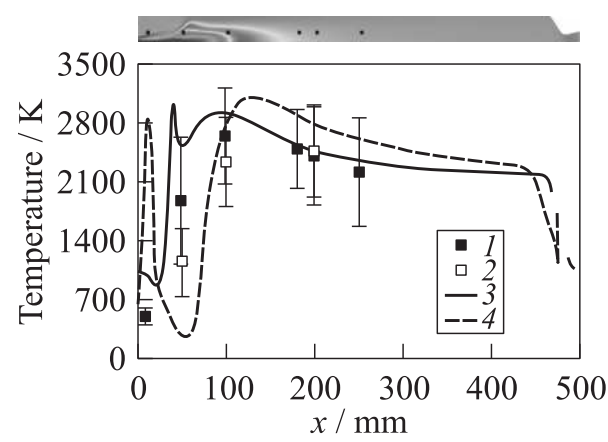

(c)

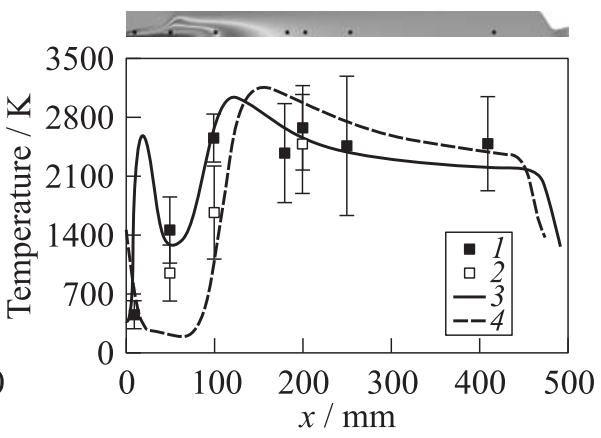

(b)

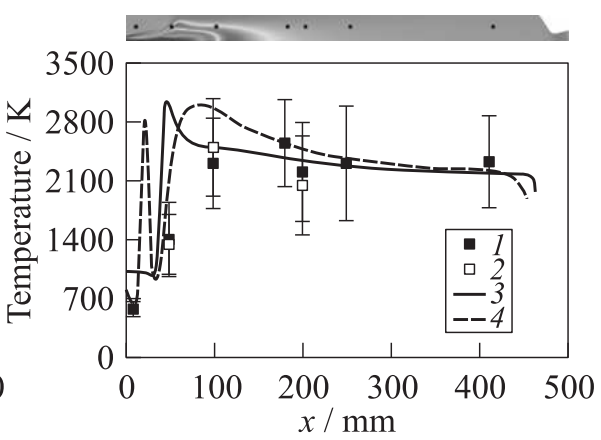

(d)

Figure 7 Comparison of Mascotte A-10 CARS temperature measurements [13, 14] ( 1 - CARS $\mathrm{H}_{2}$ and $2-\mathrm{CARS} \mathrm{H}_{2} \mathrm{O}$ ) with temperature profiles along the $x$-axis computed by Riedmann et al. [20] with RANS solvers at various $y=$ const $(z$ $=0 \mathrm{~mm})$ positions $(3-3 \mathrm{D}$ Rocflam 3 and $4-2 \mathrm{D}$-axisymmetric CFX simulation results): (a) $y=0 \mathrm{~mm}$; (b) 5 ; (c) 10; and $(d) y=15 \mathrm{~mm}$

$\mathrm{LOx}$ taper and was operated at a mixture ratio of $\mathrm{O} / \mathrm{F}=1.4$. Five years later, a second test case named C-60 was specified by Vingert et al. [22] based on a tapered coaxial injector operated at a mixture ratio of $\mathrm{O} / \mathrm{F}=2.2$. Compared to the subcritical A-10 test case, the oxygen is now in transcritical state ( $p_{\text {ox,crit }}$ $=50.4$ bar) and its disintegration can be described by a single, so-called dense gas phase accounting for real gas properties. Unfortunately, there are not many tool developers who so far simulated both the Mascotte 10- and 60-bar test cases with the same tool. Exceptions are DLR with its TAU code and Airbus DS with its in-house tool Rocflam3 and a customized version of the commercial CFD package Ansys CFX [20]. To the knowledge of the authors, [20] is the first publication which discusses the A-10 and A-60 test cases in one paper. Nevertheless, simulation results for only the Mascotte 60 -bar test case were pub- 

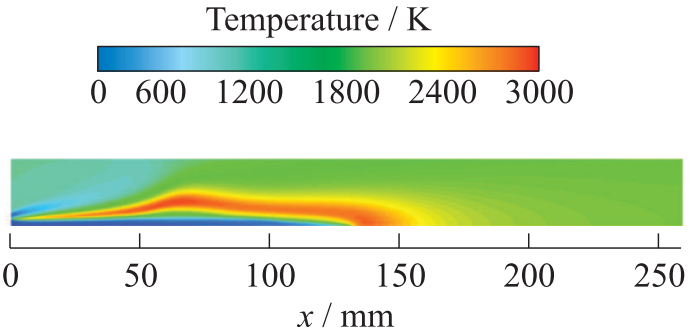

(a)

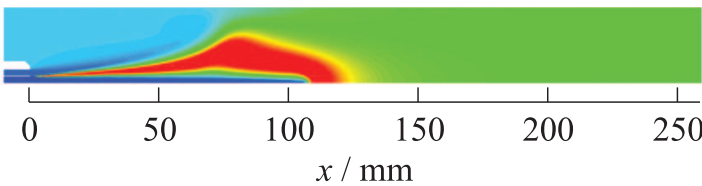

(b)

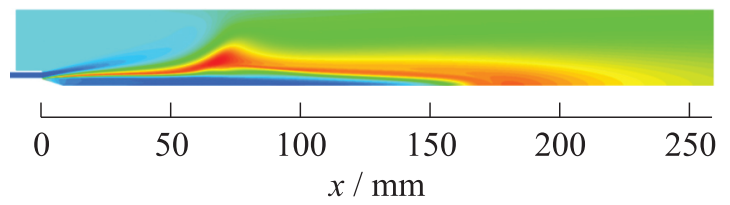

(c)
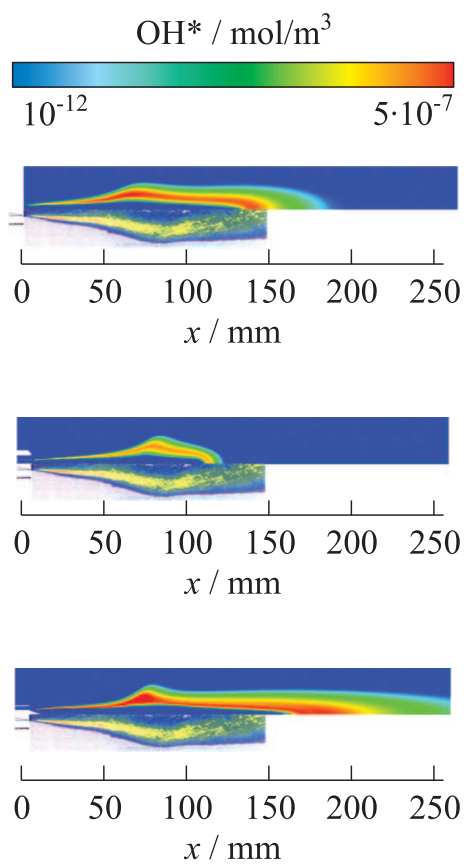

Figure 8 Comparison of Mascotte A-60 flame shapes predicted by steady RANS simulations taken from Riedmann et al. [20] (median plane images): (a) Airbus DS Rocflam3 code with a dense gas approach for the oxidizer; (b) DLR TAU-code with a real-gas-library multifluid-mixing model; and (c) CFX with a flamelet chemistry model applying supercritical oxygen parcels initialization along a cone; the experimental $\mathrm{OH}^{*}$ emission image confirms the computed flame shapes

lished by Poschner and Pfitzner in 2008 [23], Schmitt et al. in 2009 [24], and Banuti and Hannemann in 2014 [25]. All of them presented RANS solutions for A-60, except Schmitt et al. who performed a 3D LES computation for C-60. Among the RANS computations, only Riedmann et al. resolved the square duct in $3 \mathrm{D}$.

Both unsteady and steady simulations mastered the anchoring of the flame close to the injector post tip. Figure 8 compares flame shapes predicted for the A-60 test case (taken from [20]). Differences are apparent in the spreading angle of the flame and in the flame length. Unfortunately, no flame length evidence is given by the experimental data. However, the differences are not as pronounced as, perhaps, the diverse model approaches for the supercritical oxygen jet may let expect. While the DLR TAU-code used a real-gas-library multifluid-mixing model, the CFX result was obtained with a flamelet chemistry 
model applying supercritical oxygen parcels initialization along a cone and, finally, the Airbus DS Rocflam3 code employed a fairly simple dense gas inflow approach for the oxidizer. Again, three completely different tool packages with different models for turbulence, propellant disintegration, and combustion are all more or less able to reflect the experimental data. Likewise, the results achieved by Schmitt et al. with LES for test case C-60 [24] do not exhibit a much better conformance. Consequently, also the third single element test case could not confirm a superiority of unsteady high fidelity solvers over steady RANS solvers. Maybe, also, the quality of available experimental data does not permit another conclusion.

\subsection{Conclusions and Consequences from Single Element Combustor Simulations}

Considering $\mathrm{H}_{2} / \mathrm{O}_{2}$ single injector flame simulations as a first anchor point for thrust chamber flow simulations tools, the previously discussed literature review has revealed the following conclusions and consequences:

C1 RANS packages are capable to properly simulate single element combustor flows;

C2 Computational effort to resolve the unsteady 3D flow characteristic is, at least, a factor of 150 higher than 2D RANS (the factor of 10 stated by Tucker et al. [6] to account for the third direction is seen as fairly low);

C3 High grid resolution is required to resolve the unsteady flow characteristic at the injector post tip;

C4 For subcritical propellant disintegration, unsteady computations are lacking;

C5 Spray initialization in Euler-Lagrange subcritical flame computations is handled with different complexity;

C6 None of the three test cases provided flight hardware relevant conditions;

C7 There are only few tools which have demonstrated their capability to simulate cryogenic flames at sub- and supercritical pressure; and

C8 Available measurement data are often not mature enough to finally conclude on simulation capabilities (e.g., preburned gas inflow conditions, axisymmetric flame assumption in $\mathrm{OH}^{*}$ images, and CARS temperature measurements based on emission spectra of low $\mathrm{H}_{2}$ concentration signals). 
In particular, conclusions C2 and C3 question the transferability of unsteady approaches to efficient multiinjector subscale or even full-scale configurations (requirement R3). This is supported by observation $\mathrm{C} 4$ since conclusions derived from unsteady supercritical flame computations have so far not been transferred to subcritical flames (only announced but not published, see, e.g., [10]). With regard to the mission to assist and cheapen a thrust chamber design process with numerical flow analyses, also, conclusion $\mathrm{C} 7$ should be kept in mind, a capability that, e.g., the VINCI operational box requires $\left(p_{c}\right.$ over $\left.\mathrm{O} / \mathrm{F}\right)$. Consequently, in the mid-term future, only RANS solvers mastering sub- and supercritical propellant disintegration in a multiinjector configuration encourage to support the objective. Unsteady high fidelity URANS and LES solvers should, therefore, not focus on demonstrating their superiority for academic test cases (as often done) but, instead, support the evaluation and anchoring of RANS tool packages for their use in a thrust chamber design and optimization phase (as so far not done). For example, had the heat flux profile not been measured in the PennState combustor, could some (but not all) of the unsteady high fidelity simulations depicted in Fig. 3 be used to verify the RANS models shown in Fig. 4. However, this status and insight has not been reached yet. For subcrititical and transcritical $\mathrm{H}_{2} / \mathrm{O}_{2}$ flames, a comprehensive unsteady flow simulation database is even lacking.

\section{AIRBUS DS THRUST CHAMBER FLOW MODELING PHILOSOPHY}

\subsection{Propellant Disintegration Methodology}

As pointed out before, an efficient way of modeling sub-, trans-, and supercritical propellant disintegration is a key success factor for multielement combustor simulations. Grid resolution should be such that the mean features of the mixing and combustion processes are well reflected but still compatible with responsive computation times (R3). In a multiinjector configuration, this is less the exact resolution of the flame shape than the element-element interaction providing the combustion efficiency $\eta_{c *}$ and the element-wall interaction being accountable for the wall heat flux. Moreover, a 2D-axisymmetric simulation approach is desirable for configuration layout and optimization and a 3D approach is aimed for final justification. Geometrically complex grids as, e.g., with protruding solid cones or injection element internal flow resolution are CPU-intensive and not practical, for example, for injector pattern evaluation and optimization purposes, since each pattern variation requires a new mesh.

Highest flexibility is obtained by an Eulerian-Lagrangian approach, where propellant parcels are injected into a continuous gas flow. On their path through 


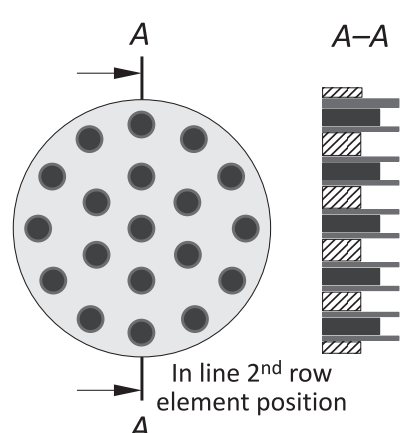

A

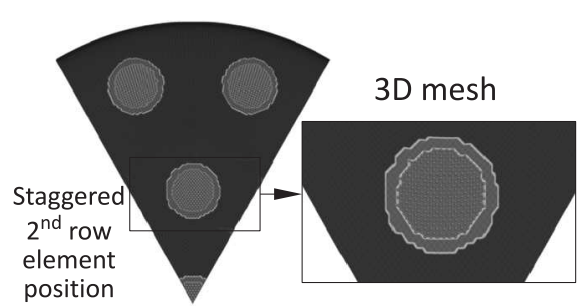

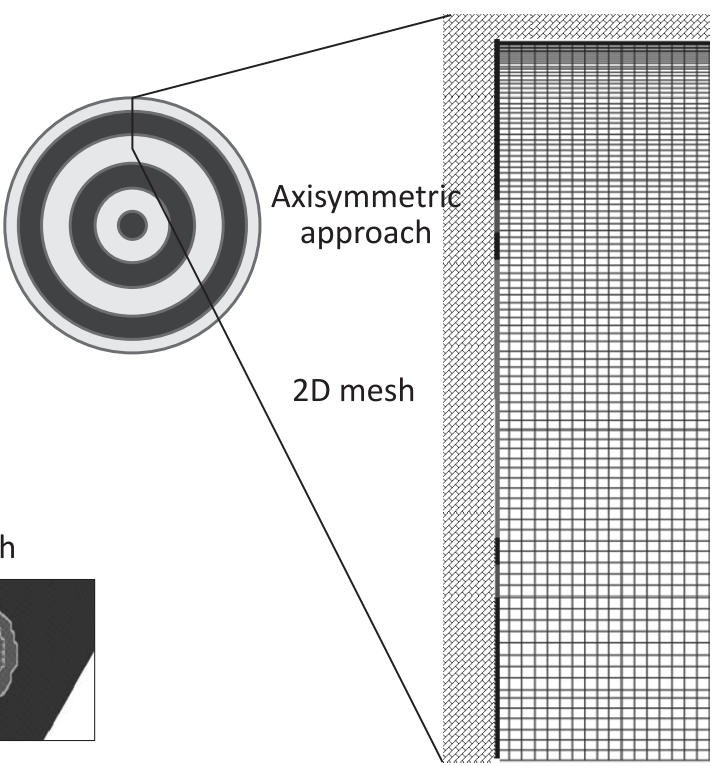

Figure 9 Airbus DS methodology of propellant disintegration simulation: 2D axisymmetric approach via annular slots maintaining radial injector dimensions (top) and 3D approach assigning propellant initialization areas on the face plate grid (bottom)

the combustion chamber, the parcels are heated up and evaporated. Source terms exchanged between dispersed propellant parcel phase and continuous gaseous phase ensure the conservation of mass, momentum, and energy. It is highlighted that propellant parcels traced by the Lagrange module can be in liquid as well as in transcritical state. For the latter, of course, no heat of evaporation for phase change is accounted. Even supercritical or gaseous propellant parcels with a virtual infinite evaporation rate can be injected, which, of course, corresponds to a simple specific mass, momentum and enthalpy source term initialization $[1,2]$.

As is illustrated in Fig. 9 for a 19-element subscale injector, the propellant parcels are initialized over the respective inflow areas at the face plate. Herewith, no continuous propellant inflow has to be resolved, i. e., the wall remains closed. This can be done on a $3 \mathrm{D}$ mesh and on a $2 \mathrm{D}$ mesh used for axisymmetric computations, where elements of a row are lumped to an annular slot. Due to the discrete injection of the propellant parcels, the actual injection velocity and momentum can be retained in a $2 \mathrm{D}$-axisymmetric simulation although the actual area is enlarged once the radial dimensions of the elements are kept. With a continuum inflow approach (gaseous or dense gas) either the cross sections of the slots have to be reduced, i. e., radial dimensions of the elements are no more 
representative, or the inflow momentum has to be reduced. More generally, also mixed inflow conditions are conceivable, where, e.g., $20 \%$ of the propellant mass flow enters as continuum and the other $80 \%$ as discrete propellant parcels [26].

Figure 9 highlights how easily an element pattern change, e.g., second row injection elements placed in between two outer row injection elements, can be simulated without changing the mesh but only adapting the propellant parcels initialization areas. Such an evaluation can, however, only be performed in three dimensions. In 2D-axisymmetric mode, nevertheless, the impact of element-row distance and element-to-wall distance can be assessed.

Airbus DS has integrated this versatile propellant disintegration methodology in its three operational design tools Rocflam-II, Rocfam3, and CFX and successfully applied to all three single element test cases discussed in section 2 (see $[11,17,18,20])$. Results have already been displayed in Figs. 4 to 8. In section 4 , the crucial transfer to multielement sub- and full-scale combustor configurations will be addressed. In case of liquid and transcritical spray initialization, of course, the droplet size and velocity distributions are free parameters. However, they can be anchored to chamber pressure and calorimetric wall heat flux measurement results since the spray is a crucial parameter for combustion efficiency (chamber pressure buildup) and wall heat flux profile (see subsection 4.1). The finer the spray, the better the combustion efficiency and the steeper the wall heat flux increase after injection.

For coaxial injector elements as commonly used in $\mathrm{LOx} / \mathrm{H}_{2}$ combustors, Airbus DS has derived spray parameters in dependence of element mass loading and mixture ratio.

\subsection{Available Tools}

Rocflam3 is a 3D spray combustion CFD tool developed by Airbus DS as successor of the 2D-axisymmetric code Rocflam-II [27]. It is a Finite-Volume solver for nonorthogonal, boundary-fitted, and block-structured grids that applies the SIMPLE (semiimplicit method for pressure-linked equations) algorithm to solve the compressible RANS equations in three spatial dimensions [28].

Rocflam3 uses a $k-\varepsilon$ turbulence model with either low-Reynolds or 2-layer approaches close to the wall. Optionally, an equilibrium or global reaction scheme based chemistry model can be employed which both account for turbulent combustion and real gas effects. A Lagrangian spray module is incorporated for propellant parcel tracking and evaporation modeling. As Rocflam-II, also, Rocflam3 pursues the simulation capability of evaporation, flow, and combustion for nearly arbitrary propellant combinations. Both the general propellant disintegration methodology described above and the equilibrium-based presumed probability 
density function (PDF) chemistry model using look-up tables do strongly support this objective.

While Rocflam-II is already a firm part of every Airbus DS thrust chamber pre- and development program (see subsections 4.2 and 4.3 ), Rocflam3 is currently in its verification phase and still has to be extended to reach the versatility of Rocflam-II.

The second numerical thrust chamber flow simulation tool that is in use at Airbus DS is the commercial CFD package Ansys CFX. Several modeling extensions have been introduced by Airbus DS to apply this high performance, general purpose 3D fluid dynamics code to rocket thrust chamber flows $[11,17]$. Two different combustion models, namely, the chemical equilibrium and the flamelet approach, have been tested. The equilibrium-based combustion model is not a CFX standard combustion model. It was developed and implemented via user defined functions and accesses the same chemistry look-up tables as Rocflam3 does. A more sophisticated model is the flamelet model because it ensures accurate chemistry kinetics and accounts for nonequilibrium effects at low computational cost. However, the CFX internal flamelet table generator has been exchanged to account for high-pressure chemistry characteristics. The influence of turbulence on combustion is treated in both models by a presumed PDF approach. The Menter-SST (shear stress transport) model is the recommended turbulence model for combustor simulations. Moreover, the CFX fluid properties data base has been extended by real gas data and sophisticated mixing rules for viscosity and heat conductivity have been implemented also via user-defined functions. By this, it is ensured that the mixing but also the subsequent combustion process are covered properly from very low up to very high temperatures.

For propellant parcel tracking, also, a Lagrangian module is available which takes special care of vanishing surface tension and evaporation enthalpy above the critical pressure.

In several former publications, simulation results of the Rocflam-II, Rocflam3, and CFX tool packages have been contrasted and discussed (see, e. g., $[11,17,20]$ ). Impacts of chemistry and turbulence models and parameters have extensively been compared and assessed by various test case simulations leading to a recommended package setup and model composition. This heritage, of course, is inherently included in the sections 2 and 4 simulation results and not discussed here. Moreover, solution grid independence as already addressed in subsection 2.1 is a critical issue in multiphase combustion flow computations. For the following sub- and full-scale rocket combustor simulations, grid independence is reached when chamber pressure and wall heat flux distribution remain unchanged. Criteria for wall and injection element resolution are derived from lab- and subscale simulations (see section 2 and subsection 4.1) and then transferred to full-scale predictions. This strategy has proven efficient in minimizing costly full-scale grid variation studies. 


\section{SIMULATION RESULTS OF SUB- AND FULL-SCALE MULTIELEMENT COMBUSTORS}

In order to generate high thrust and high combustion efficiency, a multielement injector is indispensable to disintegrate the enormous propellant mass flow rates which are fed into the combustion chamber of a rocket engine within a minimized volume $\left(l^{*}<1 \mathrm{~m}\right)$. Since usually combustion efficiency, thrust, and vacuum specific impulse at a certain mass flow rate and mixture ratio are specified to a thrust chamber developer, requirement R1 addressed in Introduction needs to be mastered. As seen before, single element combustor tests may contribute to precharacterize different injector configurations, e. g., element dimensions, and serve as first anchor point for numerical simulation tools but definitely do not contribute much to $\mathrm{R} 1$ verification. That is the reason why a rocket thrust chamber developer prefers testing a potential full-scale injection element in a multiinjector configuration. In this context, subscale testing does not mean to scale the elements down to a smaller size, but to use the full-scale element in an injector pattern that reflects the characteristics of the full-scale injector but with a reduced number of elements. The tests, of course, no more provide detailed information about the single flames but deliver the crucial characteristics of a rocket thrust chamber which are pressure buildup by combustion (corresponds to combustion efficiency) and heat loading. Hence, test results are much more comprehensive and extremely suited to validate numerical thrust chamber flow simulation tools with regard to requirements R1 and R2.

\subsection{Airbus DS 19-Element Subscale Combustor}

Along with the single element test cases, also, an $\mathrm{H}_{2} / \mathrm{O}_{2}$ subscale combustor test case has been presented at the 3rd Rocket Combustion Modeling Workshop in 2006 by Knab and Preclik [29]. The calorimetric chamber consists of 11 independently water cooled segments in the cylindrical part and 9 in the convergentdivergent nozzle section. Hydrogen and oxygen are fed through 19 Vulcain-type coaxial injector elements into the combustion chamber (Fig. 10a). The considered load point is characterized by $8.5 \mathrm{~kg} / \mathrm{s}$ propellant injection at a mixture ratio of $\mathrm{O} / \mathrm{F}=6$ providing a chamber pressure which is comparable to the reference operating point of the Vulcain rocket engine (predecessor of the Vulcain 2 engine shown in Fig. 1). Oxygen is injected in transcritical state, whereas hydrogen is injected in supercritical state. Both injection temperatures are around $T_{\mathrm{inj}} \approx 100 \mathrm{~K}$. The combustion chamber has a contraction ratio of $\varepsilon_{c}=2.5$ and a characteristic length of $l^{*}=0.84 \mathrm{~m}$. These two geometrical parameters are very similar to those of common rocket engines. Of course, 


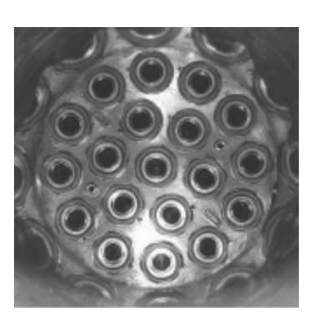

(a)

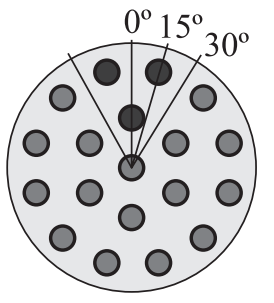

(b)

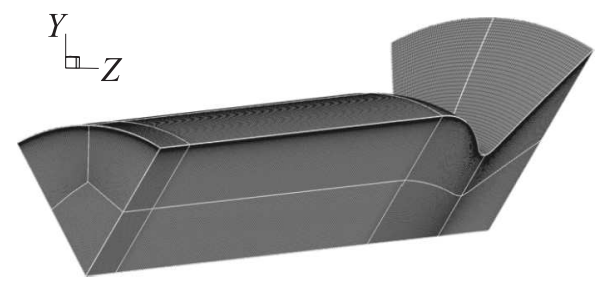

(c)

Figure 10 Airbus DS 19-element injector head (a), computational 60 degree domain with propellant injection areas $(b)$, and typical 3D structured multiblock mesh $(c)$

this is not by accident since the chamber has explicitly been designed to generate experimental results that can be transferred to full-scale rocket combustion chambers. Hence, by this test case, the tool requirements R1 and R2 can be verified at representative conditions and $\mathrm{R} 3$ can be assessed by extrapolation to full scale.

Unfortunately, apart from the industrial contributions to that workshop, there are only a few publications on this test case. Ivancic et al. [17] and Frey et al. [30] showed comparisons between 2D-axisymmetric and 3D simulations obtained with the Airbus DS in-house code Rocflam-II and the customized commercial CFX tool. Both RANS solvers provided good agreement with the experimental data. Masquelet et al. made an attempt to simulate this test case with an unsteady LES approach $[31,32]$ but did not succeed to achieve good agreement with the measured heat flux. Apparently, the unsteady high-fidelity solvers are still not prepared to adequately (not to mention efficiently) simulate a cryogenic rocket subscale chamber. This is seen as further indication that in the near-term thrust chamber, design support can only be expected from the RANS solvers.

Figure $10 b$ displays the computational grid of a 60 degree chamber segment used with symmetry boundaries in circumferential direction for a Rocflam3 simulation [26]. It is a block-structured grid with $4.2 \cdot 10^{6}$ grid cells, hence about 100 times more than in a respective 2D-axisymmetric simulation. One can see that the injection elements are not particularly resolved by the grid. Instead, the boundary cells which overlap with the injection elements are used for injection (see Fig. 9).

As the injection areas are reproduced almost exactly in three dimensions (Fig. 10c), the application of the source term approach for the hydrogen injection in 2D-axisymmetric mode is not necessary anymore. Therefore, the gaseous hydrogen enters the domain via inlet boundaries with prescribed mass flow rate. 


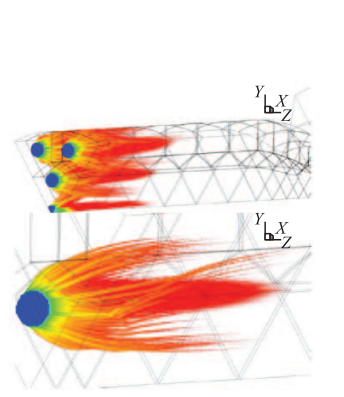

(a)

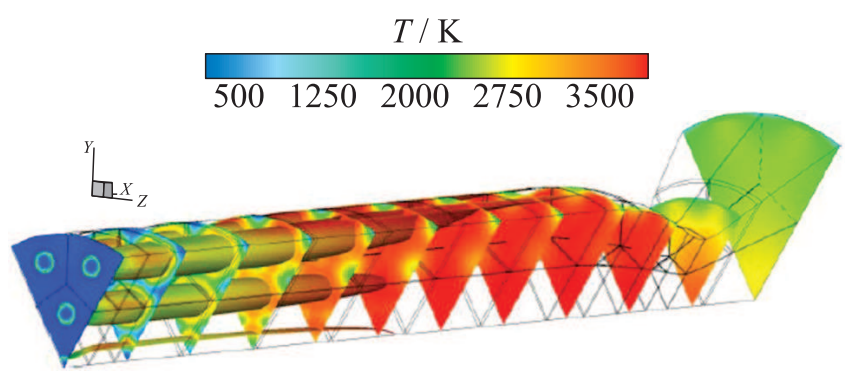

(b)

Figure 11 The 3D Rocflam3 simulation of the Airbus DS 19-element subscale combustor [26]: (a) supercritical oxygen parcel trajectories with temperature designated by color and mass indicated by point size; and $(b)$ temperature field with isosurfaces for stoichiometric mixture ratio

Although the inlet areas are not resolved exactly by the grid, the prescribed mass flow rate is achieved. However, there is a small deviation in the momentum due to that. A different approach has also been chosen for the oxygen injection: $20 \%$ of the oxidizer mass flow rate enters the domain via inlet boundaries while the remaining $80 \%$ are treated by the Lagrangian module. The number of representative parcel trajectories tracked in the 3D simulation is about 560000 which are about 22 times more than in the $2 \mathrm{D}$-axisymmetric simulation. If the complete oxidizer mass flow is treated by the Lagrangian module, the gaseous hydrogen flow is deflected at the faceplate. Hence, the mixed oxidizer inflow conditioning helps to stabilize the Rocflam3 simulation and accelerates its convergence [26].

Figure $11 a$ visualizes the supercritical oxygen parcel trajectories. The droplet temperature is designated by the color while the droplet mass is indicated by the size of the points in the scatter diagram. The smallest droplets are not visible due to that scaling.

Figure $11 b$ shows several slices at distinct axial positions for the temperature as well as the isosurfaces for the stoichiometric mixture ratio. It gives the first impression of the 3D flow field which Rocflam3 computes for the 19element subscale combustion chamber. From the $\mathrm{O} / \mathrm{F}=8$ isosurfaces, one can see that the flames emanating from the outer injector row gradually approach the liner wall. This behavior has a noticeable effect on the wall heat flux.

Figure $12 a$ displays the specific wall heat flux profiles for each of the 96 grid cell rows in circumferential direction together with the resulting mean heat flux. 


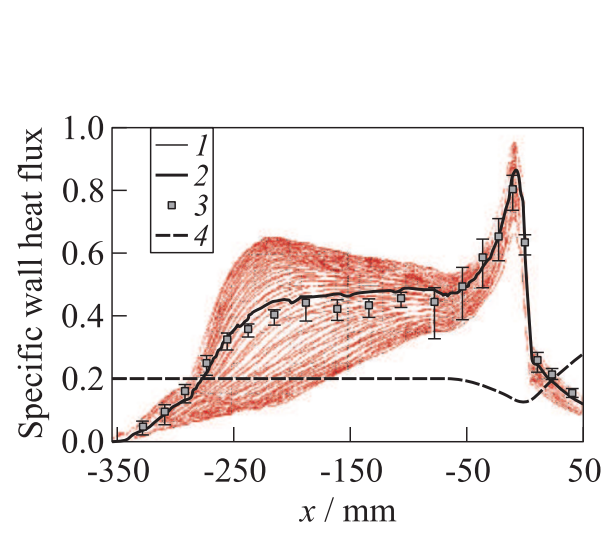

(a)

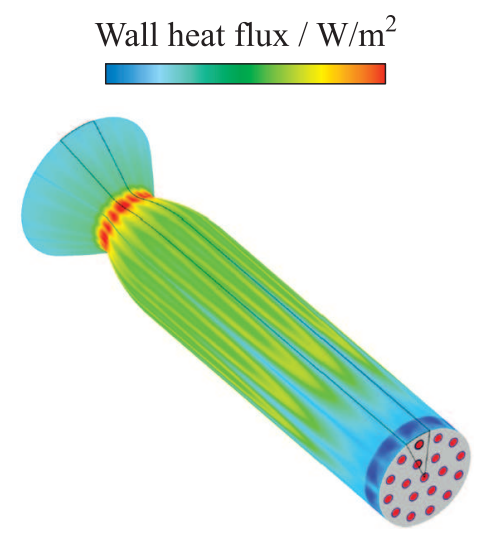

(b)

Figure 12 Specific wall heat flux profiles computed with Rocflam3 along with calorimetric measurement data [26] (a) (1 - local heat flux; 2 - mean heat flux; 3 - test data; and 4 - contour) and 3D heat flux distribution simulated with CFX [17] (b)

Also, the depicted 3D CFX test case computation (Fig. 12b) approves the pronounced 3D heat flux distribution [17]. Especially in the middle of the cylindrical part, the circumferential variation of the wall heat flux is considerable. At $x=-220 \mathrm{~mm}$, one can detect that the curves with the highest heat flux values suddenly decrease. This can also be seen from the 3D CFX surface plot. Here, overstoichiometric gas compositions occur locally close to the wall. As expected, the circumferential variation decreases in downstream direction. The mean heat flux values fit almost perfectly the experimental data. Consequently, the tool capability to master R2 has been demonstrated. At this place, it is highlighted that the wall heat flux in such a high-pressure combustor environment reaches values up to $100 \mathrm{MW} / \mathrm{m}^{2}$ and is, hence, no more predictable by models merely tuned to smooth wall, flat plate boundary layer-like flows. The models need to account for wall roughness which over time evolves differently along the chamber wall depending on local wall temperature levels, i. e., local wall heat flux and cooling efficiency [33].

Since in the computation a propellant mass inflow rate of $8.5 \mathrm{~kg} / \mathrm{s}$ has been prescribed via the boundary cells, the calculated total chamber pressure is a measure for the simulated combustion completeness or combustion efficiency. Rocflam 3 computes a total chamber pressure of $p_{c}=97.8$ bar at the throat corresponding to a combustion efficiency of $\eta_{c^{*}}=98.8 \%$. This is in close agreement with the measured chamber pressure and impressively confirms the Rocflam3 capability to master also requirement R1. 


\subsection{Vulcain 2 Combustion Chamber}

The identical propellant disintegration methodology is then used in a $3 \mathrm{D}$ Rocflam3 simulation of the Vulcain 2 combustion chamber. From the more than 500 injection elements, 29 are considered in a 14.4 degree segment and resolved with $23 \cdot 10^{6}$ grid cells. Notice that this is a factor of 5.5 more than in the previous subscale simulation, however, maintaining its resolution. Vulcain 2 burns $310 \mathrm{~kg} / \mathrm{s}$ of supercritical hydrogen and transcritical oxygen at a mixture ratio of 7.2. Figure $13 a$ displays the $3 \mathrm{D}$ temperature field within the combustion chamber and Fig. $13 b$ compares it with a $2 \mathrm{D}$-axisymmetric Rocflam-II simulation. One can clearly see the $3 \mathrm{D}$ resolution of the injection element pattern. Both in two and three dimensions, the flames are anchored close to the face plate. Elements of the 3D pattern which are cut by the cross section in their symmetry line exhibit a similar flame structure as the $2 \mathrm{D}$ axisymmetric simulation does. Also, the distance from the face plate where the full combustion temperature is reached is predicted almost identically. For Rocflam-II, a coupling with a $3 \mathrm{D}$ conjugate heat transfer analysis of the cooling circuit flow is performed providing the axial wall temperature distribution. Hot gas side and cooling circuit computations are performed sequentially up to convergence of the wall temperature distribution [2]. Usually, 5 to 10 coupling loops are sufficient, hence enhancing the computational effort about $10 \%$ to $50 \%$ depending on the resolution effort spent in the cooling channel model. For the 3D Rocflam 3 simulation, the Rocflam-II wall temperature profile has been imposed in axial direction and prescribed as uniform in circumferential direction.

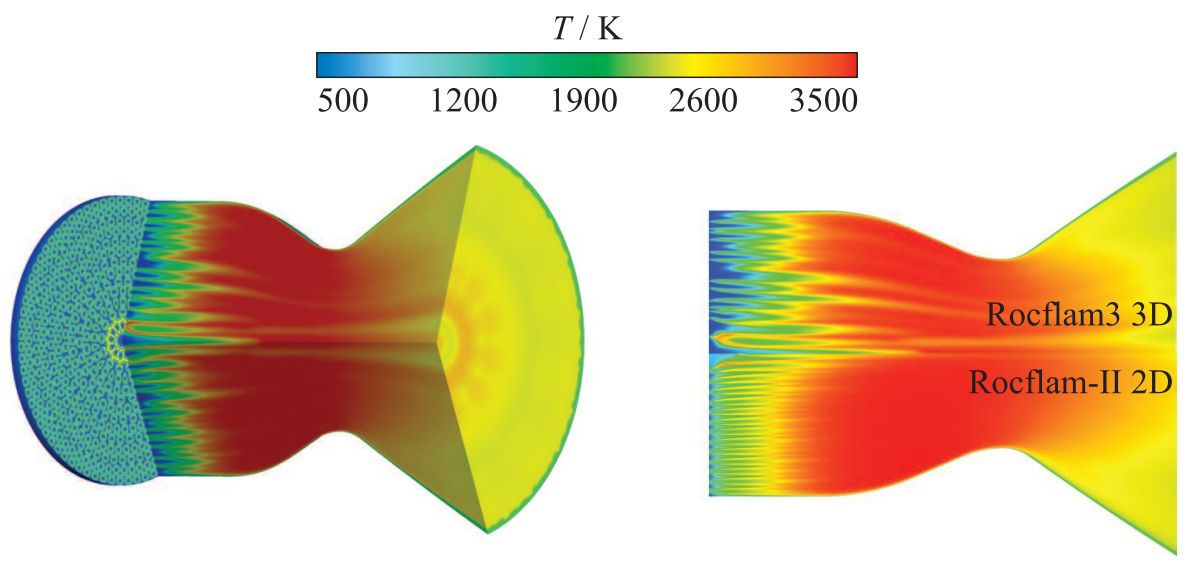

Figure 13 The 3D Rocflam3 simulation of the Vulcain 2 combustion chamber flow $(a)$ and comparison of the temperature field with a 2D-axisymmetric Rocflam-II computation $(b)$ 
At this place, it is mentioned that such 3D full-scale rocket combustion chamber flow computations are still not state of the art and can be seen as the result of more than 25 years of continuous effort in thrust chamber modeling at Airbus DS. To integrate the results of such sophisticated steady RANS solutions into the design, justification process of a new hardware will be the challenge of the coming years if they contribute to reduce development time and cost depends on the validation level and added value reached. With the conclusions drawn in subsection 2.3, 3D URANS and LES solvers are even not believed to accomplish that in the mid-term. As it is shown in the next subsection, 2D-axisymmetric RANS flow simulations have already reached this status.

\subsection{Aestus Thrust Chamber}

As shown in Fig. 1, launcher propulsion as well as trajectory-attitude control systems burning hypergolic fuels still constitute an important part of rocket propulsion systems. Up to flight qualification of the cryogenic upper stage engine VINCI, the Airbus DS Aestus engine is the only reignitable upper stage engine for Ariane. It burns $8.8 \mathrm{~kg} / \mathrm{s}$ of the storable propellants MMH/NTO at a mixture ratio of $\mathrm{O} / \mathrm{F}=2.05$. Consequently, a numerical thrust chamber simulation tool must have the capability to handle two liquid propellant phases (phenomenon P2).

Figure 14 outlines how the MMH and NTO propellant parcels are initiated to reflect the combined swirl-jet injector elements in a 2D-axisymmetric Rocflam-II

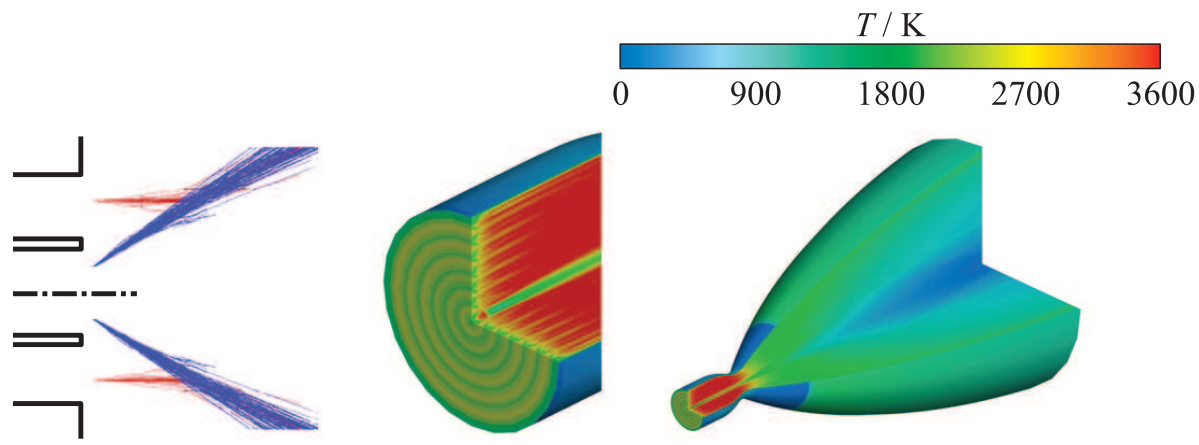

Figure 14 Simulation of the Aestus thrust chamber with regeneratively cooled combustion chamber and radiatively cooled nozzle extension burning the storable propellant combination $\mathrm{MMH} / \mathrm{NTO}$ : bipropellant parcels tracking and spray initialization approaching the injector element characteristics in a $2 \mathrm{D}$-axisymmetric computation 
simulation. Since the combustion chamber is regeneratively cooled with MMH, a coupling with a conjugate heat transfer analysis of the coolant channel flow is established to determine the internal combustion chamber wall temperature distribution (phenomenon P4) [34].

For the Aestus engine test and flight data for integral chamber heat load, nozzle wall temperature distribution, chamber pressure, and vacuum specific impulse are available. The agreement between test data and prediction with a deviation below $1 \%$ is excellent for the heat load. Regarding the chamber pressure, i. e., combustion efficiency, the simulation slightly underestimates the measured value by less than $0.5 \%$. Consequently, the applied model reflects the thermochemical phenomena evaporation, mixing, and combustion, which are responsible for the simulated chamber pressure buildup, fairly well. Finally, also the vacuum specific impulse is underestimated by $0.9 \%$ compared to flight data. This indicates that the $\mathrm{MMH} / \mathrm{NTO}$ combustion kinetics in sub- and supersonic flow regimes are still not perfectly captured (notice that 1 percent deviation in vacuum specific impulse is around $3 \mathrm{~s}$ ). Compared to [34], the mechanism has further been upgraded and is still under continuous improvement. A simulation tool fulfilling the requirements $\mathrm{R} 1, \mathrm{R} 2$, and $\mathrm{R} 3$ in such a way can, of course, beneficially be used for further storable engine developments as it is currently under way at Airbus DS with a 5-kilonewton engine.

\section{CONCLUDING REMARKS}

Numerical rocket thrust chamber flow simulation is seen by various parties as a forthcoming contribution to reduce test and design efforts and, thereby, development time and cost for such high-tech devices. However, the view to configure the tools with useful solvers and thermochemical models is completely different. Knowing that it is still a challenge for steady RANS solvers to serve this objective, more and more unsteady high fidelity solver developers use single-element combustor simulations to demonstrate their superiority. The paper has outlined that such an approach is not target-oriented with regard to the development of a thrust chamber design supportive tool. Instead of competing for prevalence, it is proposed to complement each other.

In particular, the multielement injector configurations of sub- and full-scale combustors require efficient and propellant state considering propellant disintegration modeling approaches. These approaches have to be transferred together with grid resolution requirements and thermochemical model parameter settings from single- to multielement combustor simulations. Supported by the literature review addressed in this paper, it is concluded subsequently why in the mid-term only steady RANS solvers can succeed in this task. However, URANS or LES solvers could support them on their mission: 
- so far, only steady RANS simulations have demonstrated their ability to simulate laboratory-, sub, and full-scale thrust chamber flows;

- almost no sub- and full-scale multielement combustor simulations with unsteady flow solvers are available and expectable that deliver design supportive information in typical design time frames (e.g., 1-3 days in conceptual phase, 1-3 weeks in design and optimization phases, 1-3 months in justification phase);

- steady 2D-axisymmetric simulations can contribute to thrust chamber layout and optimization purposes, whereas unsteady 2D-axisymmetrical simulations of an inherently 3D unsteady flow appear questionable;

- the prediction capability of sub- and supercritical propellant disintegration in LOx/hydrocarbons, LOx/hydrogen, or MMH/NTO propulsion systems requires the computation intensive validation of a variety of models and model parameters; and

- modeling assumptions used in steady RANS tools to describe propellant disintegration, turbulent mixing, and combustion as well as heat transfer could be improved and anchored by detailed unsteady high fidelity simulations of dedicated laboratory-scale test cases.

Since the latter has up to now not been conducted for the three single-element combustor test cases addressed in this paper, the authors see the potential and mission of the unsteady high fidelity solvers there.

\section{ACKNOWLEDGMENTS}

Part of this work was performed within the national technology program TARES. This program is sponsored by the German Space Agency, DLR Bonn, under contract No.50RL1210. Lively discussions held on the paper's subject within the framework of the national research program SFB/TRR-40 are gratefully acknowledged.

\section{REFERENCES}

1. Knab, O., A. Fröhlich, J. Görgen, and D. Wiedmann. 2002. Advanced thrust chamber layout tools. 4th Conference (International) on Launcher Technology - Space Launcher Liquid Propulsion. Liege, Belgium.

2. Knab, O., M. Frey, J. Görgen, C. Mäding, K. Quering, and D. Wiedmann. 2009. Progress in combustion and heat transfer modelling in rocket thrust chamber applied engineering. AIAA Paper No. 2009-5477. 
3. Marshall, W., S. Pal, R. Woodward, and R. Santoro. 2005. Benchmark wall heat flux data for a $\mathrm{GO}_{2} / \mathrm{GH}_{2}$ single element combustor. AIAA Paper No. 2005-3572.

4. Habiballah, M., L. Vingert, J. Traineau, and P. Vuillermoz. 1996. Mascotte: A test bench for cryogenic combustion research. 47th Astronautical Congress (International). Beijing, China.

5. Pal, S., W. Marshall, R. Woodward, and R. Santoro. 2006. Test case RCM 1: PennState preburner combustion - wall heat flux data for a $\mathrm{GO}_{2} / \mathrm{GH}_{2}$ uni-element combustor. 3rd Workshop (International) on Rocket Combustion Modeling Proceedings. Paris.

6. Tucker, K., S. Menon, C. Merkle, J. Oefelein, and V. Yang. 2008. Validation of high fidelity CFD simulations for rocket injector design. AIAA Paper No. 2008-5226.

7. Sozer, E., E. Hassan, S. Yun, S. Thakur, J. Wright, M. Ihme, and W. Shyy, 2010. Turbulence-chemistry interaction and heat transfer modeling of $\mathrm{H}_{2} / \mathrm{O}_{2}$ gaseaus injector flows. 48th AIAA Aerospace Sciences Meeting. Orlando, FL.

8. Daimon, Y., H. Negishi, and N. Yamanishi. 2010. Combustion and heat transfer modeling in regeneratively cooled thrust chambers (Wall Heat Flux Validation). 46th AIAA/ASME/SAE/ASEE Joint Propulsion Conference \& Exhibit. Nashville, TN.

9. Lian, C., and C. Merkle. 2010. Contrast between steady and time-averadged unsteady combustion simulations. AIAA Paper No. 2010-371.

10. Lempke, M., P. Gerlinger, M. Aigner, and M. Rachner. 2011. Steady and unsteady RANS simulations of cryogenic rocket combustors. AIAA Paper No. 2011-101.

11. Ivancic, B., H. Riedmann, M. Frey, O. Knab, S. Karl, and K. Hannemann. 2016. Investigation of different modeling approaches for computational fluid dynamics simulation of high-pressure rocket combustors. Progress in propulsion physics. Eds. M. Calabro, L. DeLuca, S. Frolov, L. Galfetti, and O. Haidn. EUCASS advances in aerospace sciences book ser. TORUS PRESS - EDP Sciences. 8:95-116.

12. Vingert, L., and M. Habiballah. 2001. Test case RCM-2: Mascotte single injector 10 bar. 2nd Workshop (International) on Rocket Combustion Modeling - Atomization, Combustion and Heat Transfer Proceedings. Lampolshausen.

13. Candel, S., G. Herding, R. Snyder, P. Scouflaire, C. Rolon, L. Vingert, M. Habiballah, F. Grisch, M. Pealat, P. Bouchardy, D. Stepowski, A. Cessou, and P. Colin. 1998. Experimental investigation of shear coaxial cryogenic jet flames. J. Propul. Power 14(5):826-834.

14. Grisch, F., P. Bouchardy, and W. Clauss. 2003. CARS thermometry in high pressure rocket combustors. Aerosp. Sci. Technol. 7(4):317-330.

15. Nicole, A., G. Ordonneau, and M. Theron. 2009. 3D simulation of $\mathrm{LOx} / \mathrm{GH}_{2}$ Mascotte test case at 10 bar. 3rd European Conference for Aeronautics and Space Sciences. Versailles, France.

16. Izard, J., and A. Mura. 2011. Lagrangian modeling of turbulent spray combustion: Application to rocket engine cryogenic conditions. Progress in propulsion physics. Eds. L. DeLuca, C. Bonnal, O. Haidn, and S. Frolov. EUCASS advances in aerospace sciences book ser. TORUS PRESS-EDP Sciences. 2:207-224. 
17. Ivancic, B., H. Riedmann, and M. Frey. 2012. Validation of turbulent combustion models for 3D-simualtions of liquid $\mathrm{H}_{2} / \mathrm{O}_{2}$ rocket combustors. Space Propulsion Conference. Bordeaux, France.

18. Riedmann, H., B. Kniesner, M. Frey, and C.-D. Munz. 2013. Numerical investigation of spray combustion and flow in $\mathrm{LOx} / \mathrm{H}_{2}$ subscale rocket combustors. 5th European Conference for Aeronautics and Space Sciences. Munich, Germany.

19. Farmer, R., R. Pike, and G. Cheng. 2005. CFD analyses in complex flows. Comput. Chem. Eng. 29(11-12):2386-2403.

20. Riedmann, H., D. Banuti, B. Ivancic, O. Knab, and K. Hannemann. 2015. Modeling of $\mathrm{H}_{2} / \mathrm{O}_{2}$ single-element rocket thrust chamber combustion at sub- and supercritical pressures with different CFD tools. 6th European Conference for Aeronautics and Space Sciences. Krakow, Poland.

21. Habiballah, M., and S. Zurbach. 2001. Test case RCM-3, Mascotte single injector. 2nd Workshop (International) on Rocket Combustion Modeling Proceedings. Lampoldshausen.

22. Vingert, L., A. Nicole, and M. Habiballah. 2006. Test case RCM-2, Mascotte single injector. 3rd Workshop (International) on Combustion Modeling Proceedings. Paris.

23. Poschner, M., and M. Pfitzner. 2008. Real gas CFD simulation of supercritical $\mathrm{H}_{2}$ LOx combustion in the Mascotte single-injector combustor using a comercial CFD code. AIAA Paper No. 2008-952.

24. Schmitt, T., L. Selle, B. Cuenot, and T. Poinsot. 2009. Large-eddy simulation of transcritical flows. C.R. Acad. Sci. II B Mec. 337:528-538.

25. Banuti, D., and K. Hannemann. 2014. Application of a real-gas-library multi-fluidmixing model to supercritical sinngle injector flow. 50th AIAA/ASME/SAE/ASEE Joint Propulsion Conference. Cleveland, OH.

26. Riedmann, H., B. Kniesner, M. Frey, and C. D. Munz. 2014. 3D modeling of spray combustion and flow in a $40 \mathrm{kN} \mathrm{H}_{2} / \mathrm{O}_{2}$ subscale rcket trust camber. Space Propulsion Conference. Cologne, Germany.

27. Frey, M., B. Kniesner, and O. Knab. 2011. Consideration of real gas effects and condensation in a spray combustion rocket-thrust-chamber design tool. Progress in propulsion physics. Eds. L. DeLuca, C. Bonnal, O. Haidn, and S. Frolov. EUCASS advances in aerospace sciences book ser. TORUS PRESS - EDP Sciences. 2:285296.

28. Riedmann, H., B. Kniesner, M. Frey, and C.-D. Munz. 2014. Modeling of combustion and flow in a single element $\mathrm{GH}_{2} / \mathrm{GO}_{2}$ combustor. CEAS Space J. 6(1):47-59. doi: 10.1007/s12567-013-0056-3.

29. Knab, O., and D. Preclik. 2006. RCM test-case RCM-3: "EADS-ST subscale chamber." 3rd Workshop (International) Rocket Combustion Modeling Proceedings. Paris.

30. Frey, M., T. Aichner, B. Ivancic, B. Kniesner, and O. Knab. 2010. Modeling of rocket combustion devices. 10th AIAA/ASME Joint Thermophysics and Heat Transfer Conference. Chicago, IL. 
31. Masquelet, M. 2006. Simulations of a sub-scale liquid rocket engine: Transient heat transfer in a real gas environment. Atlanta, GA: Georgia Institute of Technology. Master Thesis.

32. Masquelet, M., S. Menon, Y. Jin, and R. Friedrich. 2009. Simulation of unsteady combustion in a $\mathrm{LOx}-\mathrm{GH}_{2}$ fueled rocket engine. Aerosp. Sci. Technol. 13:466-474.

33. Kniesner, B., M. Frey, und O. Knab. 2013. Consideration of wall roughness effects in the prediction of heat transfer in rocket combustion chambers. 5th European Conference for Aeronautics and Space Sciences. Munich, Germany.

34. Aichner, T., H. Riedmann, B. Kniesner, and O. Knab. 2012. Development of an advanced global MMH-NTO combustion model for CFD application. Space Propulsion Conference. Bordeaux, France. 